\title{
Dauernd verstopft? Das hilft!
}

\section{Hier steht eine Anzeige.}

\section{Der selektive Serotonin-5-HT4-Rezeptoragonist Prucaloprid lässt sich effektiv und sicher bei chroni- scher Obstipation einsetzen - auch bei älteren Menschen.}

Chronische Obstipation verursacht gravierende Beschwerden und schränkt die Lebensqualität deutlich ein. Etwa 15\% der Bevölkerung leiden darunter, vor allem ältere Menschen. So sind Frauen jenseits des 65. Lebensjahres zu 26\%, Männer zu $16 \%$ betroffen. Bei den über 84 -Jährigen ist es knapp ein Drittel, bei Bewohnern von Pflegeheimen sind es bis zu $80 \%$, erläuterte Professor Martin Wehling vom Institut für experimentelle und klinische Pharmakologie und Toxikologie Mannheim der Universität Heidelberg beim GastroenterologenKongress in Hamburg.

\section{Neue S2k-Leitlinie im Anmarsch}

Demnächst soll die interdisziplinär erstellte S2k-Leitlinie zur „Epidemiologie, Pathophysiologie, Diagnostik und Therapie der chronischen Obstipation" veröffentlicht werden. In diese Leitlinie, die unter Schirmherrschaft der DGNM (Deutsche Gesellschaft für Neurogastroenterologie und Motilität) und DGVS (Deutsche Gesellschaft für Verdauungs- und Stoffwechselkrankheiten) erstellt wurde, hat auch der selektive Serotonin5-HT4-Rezeptoragonist Prucaloprid als Therapieoption Eingang gefunden.

Der Wirkstoff lässt sich effektiv und sicher bei chronischer Obstipation einsetzen, auch bei älteren Menschen. In einer Placebo-kontrollierten Untersuchung bei 300 über 65-jährigen Patienten erhöhte Prucaloprid nicht nur die Stuhlgangfrequenz, sondern reduzierte auch abdominelle Symptome und verbesserte die Lebensqualität.

Gleichzeitig erwies sich Prucaloprid auch in dieser Patientengruppe als sicher und gut verträglich. Zusätzlich zeigte eine Sicherheitsstudie an 89 älteren Patienten, dass es in Tagesdosen bis zu 2 mg nicht zu einer Verlängerung der QT-Zeit kommt.

\section{Bei Transitstörungen nach dem}

\section{Stufenschema handeln}

Danach sollte bei chronischer Obstipation als Folge einer Transitstörung nach einem Stufenschema behandelt werden, beginnend mit Allgemeinmaßnahmen und der Einnahme von Ballaststoffen. An zweiter Stelle steht die medikamentöse Therapie mit osmotisch wirksamen Substanzen, bei Verträglichkeit auch mit Lactulose, Sorbitol sowie Bisacodyl und Natriumpicosulfat.

Wird damit kein oder kein zufriedenstellender Erfolg erreicht, sollten laut Leitlinie Prokinetika vom Typ der 5-HT4-Agonisten wie Prucaloprid zum Einsatz kommen.

\section{Springer}

